$$
\text { CERN-ECP } 91-34
$$

\title{
THE SCI-BASED DATA ACQUISITION ARCHITECTURES
}

J.A.C. Bogaerts (spokesman), R. Divià, H. Müller and J.F. Renardy $\left.{ }^{*}\right)$

CERN, Geneva, Switzerland

\begin{abstract}
The scalable coherent interface (SCI) is an IEEE proposed standard (P1596) for interconnecting multiprocessor systems. The standard defines point-to-point connections between nodes, which can be processors, memories or I/O devices. Networks containing a maximum of $64 \mathrm{~K}$ nodes with a bandwidth of $1 \mathrm{Gbyte} / \mathrm{s}$ between nodes, may be constructed. An SCI is an attractive candidate to serve as a backbone for high speed, large volume data acquisition systems such as required by future experiments at the proposed Large Hadron Collider (LHC) at CERN. Work has started to simulate SCI-based architectures for data acquisition systems. The simulation program proved to be a useful tool to study SCI systems. First results are reported on a model of a large LHC experiment containing over 1000 nodes.
\end{abstract}

Presented at the Real Time'91 Conference Aachen, Germany, 12-15 March 1991

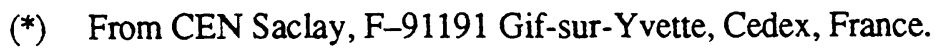




\section{THE SCI BASICS}

\subsection{Physical logical layer}

The SCI standard [1] defines point-to-point connections between nodes which can be processors, memories or I/O devices. Each node has an input port and an output port. The basic system configuration, a ringlet, consists of nodes connected by unidirectional links. A large SCI system consists of several ringlets which communicate through bridges or switches. Throughput, latency and fault tolerance can be optimized by choosing a suitable topology.

Initially, the physical connection will be based on flat cables or optical fibres. The cable transmits at a speed of 1 Gbyte/s using 16 pairs of ECL differential signal lines clocked at $250 \mathrm{MHz}$. The distance between neighbouring nodes is limited to tens of metres. The optical fibre companion standard can be used for larger distances at a reduced performance of $1.25 \mathrm{Gbits} / \mathrm{s}$. Modules and crates follow IEEE 1301 and IEEE 1301.1 standards.

On an SCI link, 16-bit symbols are transmitted synchronously from node to node. These symbols can be either part of information packets or idle symbols which carry flow control information used by the bandwidth allocation protocols. A packet contains target address (16 bits of identifier plus 48 bits of internal address), command, source address (16 bits) and control followed by $0,16,64$ or 256 bytes of data and a CRC check sum. New packets can be inserted between circulating packets by a sending node, subject to bandwidth allocation protocols which may restrict access to the ringlet for flow control purposes. Packets are removed from the ring by the destination, which transmits a small echo packet and replaces removed symbols by idle symbols. Echoes are used to signal status information (busy, CRC error, ...). This may result in retransmission of packets. Queue acceptance protocols guarantee that packets rejected by the destination will eventually be accepted. Likewise, bandwidth allocation protocols ensure that packets can eventually be transmitted.

Most SCI transactions are split in two subactions: a request and a response packet. In a write transaction, data are contained in the request packet, in a read transaction they arc in the response packet. The exception is the move transaction, which is a write-tomemory without a response subaction. Move transactions may be particularly efficient for moving data across a network, since there is no end-to-end protocol (echoes are local to ringlets) at the expense of slightly reduced error notification. Move transactions arc attractive for data acquisition purposes if large amounts of data have to be shifted.

More complex transactions arc defined, for example, lock and cache coherence primitives. Lock transactions arc intended for synchronization purposes. The SCI uses an innovative directory-based mechanism to implement cache coherence protocols. The 
directory is organized as a distributed linked list with one entry for each memory line of 64 bytes. Data may be cached coherently, locally or not at all. The standard defines a minimal cache protocol which is guaranteed to interwork with optional extensions.

\subsection{Connectivity}

The possibility to use SCIs as an interconnect to buses is a major attraction for many early applications. A subgroup (IEEE P1596.1) has been formed to define a standard bridge to VMEbus. Bridges to Futurebus+ and Fastbus are also under investigation.

Large rings are not scalable (sect. 4); several interconnected ringlets with at most $\approx 20$ nodes should be used. The SCI bridges may be constructed from two SCI node interface chips, connected back-to-back. Such bridges buffer a complete packet before data can be forwarded. More efficient bridges which can start transmission just after the target address has been received, require a different implementation on silicon.

Large systems containing many bridges may become bandwidth limited. A long path between nodes leading through bridges introduces latencies which reduce the useful processor bandwidth. Active switches providing a direct connection between any two ringlets can vastly improve system performance. Only architectures with bridges have been considered in the following sections.

\subsection{Status}

Draft 1.00 of the P1596 SCI specification has been approved by the balloting body of the IEEE Technical Committee on Microprocessors and Microcomputers. It is expected that the final document will be submitted to the IEEE Standards Board during the autumn of 1991.

The first SCI chip set consists of an SCI node interface chip and a cache and memory controller chip with an MC88110 compatible bus. These, and a VME bridge are under development by Dolphin Server Technology AS, Oslo, Norway. The SCI interface chip will transmit and receive at $1 \mathrm{Gbyte} / \mathrm{s}$, support SCI transactions with packet sizes up to 80 bytes, provide fun support for cache coherency (the "typical" configuration as proposed in the standard) with 64 byte cache lines and registers implemented following the IEEE P1212 CSR standard. The optical "G link" chip set from Hewlett Packard Inc., Palo Alto, USA, is expected to provide an SCI compatible fibre implementation at $100 \mathrm{Mbytes} / \mathrm{s}$. The first silicon should be available soon after the standard has been accepted.

Simulation of SCI has started at the Department of Informatics, University of Oslo, Norway and at CERN. The group at Oslo University is implementing a synchronous 
simulation, which operates at the SCI symbol level (resolution of $2 \mathrm{~ns}$ ), specifically aimed at simulating cache coherency and verification of the protocols. The CERN simulation is asynchronous and operates at the SCI packet level (typical resolution $\approx 100 \mathrm{~ns}$ ). It is targeted at the simulation of large SCI systems.

\section{AN SCI FOR DATA ACQUISITION}

\subsection{Connectivity}

Due to the size of detectors used in HEP experiments data acquisition systems need to be distributed over distances of $\approx 100 \mathrm{~m}$. Electronics are typically housed in hundreds of Fastbus or VMEbus crates. These systems use hundreds of embedded microprocessors and are connected to a network of workstations. Processors are used to transport filter and record detector data. Fastbus, which was specifically designed for HEP applications, can interconnect a large number of crates. For VMEbus, several ad hoc solutions were adopted before the "vertical" VICbus emerged as a standard [2]. The need for noiseimmune signal transmission has lead to the development of various optical point-to-point links.

For LHC experiments these solutions may no longer be adequate. A uniform, fast, reliable connection supported by industry is needed. The SCI provides such a solution, both on electrical and optical media. It offers a uniform interface, a speed increase of one order of magnitude and a robust protocol which includes flow control and error recovery for data transmission even over long distances.

\subsection{Data rates}

Expected data rates for future LHC experiments have been estimated in [3]. Events of 10 Gbytes are stored in pipelines at a rate of $66 \mathrm{MHz}$. After hardware filtering and zero suppression, a data stream of 1 Gbyte/s is further reduced by digital processors to be finally recorded at a rate of $\approx 100 \mathrm{Mbytes} / \mathrm{s}$. An SCI is a good candidate to handle the data flow after filtering. It is expected that LHC data acquisition systems will be able to use switch-based architectures.

\subsection{Innovations simplify systems}

The SCI has several innovative features which may simplify data acquisition systems.

With SCI it is possible to process data residing in distributed front-end memories. Data copying can be avoided and caching speeds up repeated access. The shared-memory model allows access to all data without using $\mathrm{I} / \mathrm{O}$ instructions. Use of native memory instructions is efficient for both the processor and the applications written in high-level 
languages. This avoids the need for library routines (such as the NIM Standard Routines for Fastbus) or I/O drivers (VMEbus, though memory mapped, often relies on drivers and interrupts to access data).

The SCI provides coherent access to shared data through caches. In traditional data acquisition systems, synchronization between processors is achieved by exchanging messages, often using a bus which has been optimized for transport of large blocks of data. This communications software is notoriously slow and difficult to implement.

Trigger processors typically need only random access to a fraction of the data which is scattered in many front-end memories. The SCI is particularly effective in accessing small amounts of data distributed over a large system.

A difficult problem (event building) as illustrated in fig. 1, may more easily be implemented using a shared-memory model and memory mapping. At present, much of the processing is being done using embedded microprocessors, which rarely use virtual memory. Future data acquisition systems will use Real Time Operating Systems (RTOS) which support virtual memories and caches. The SCI is especially well suited to interconnect processors and memories with this architecture.

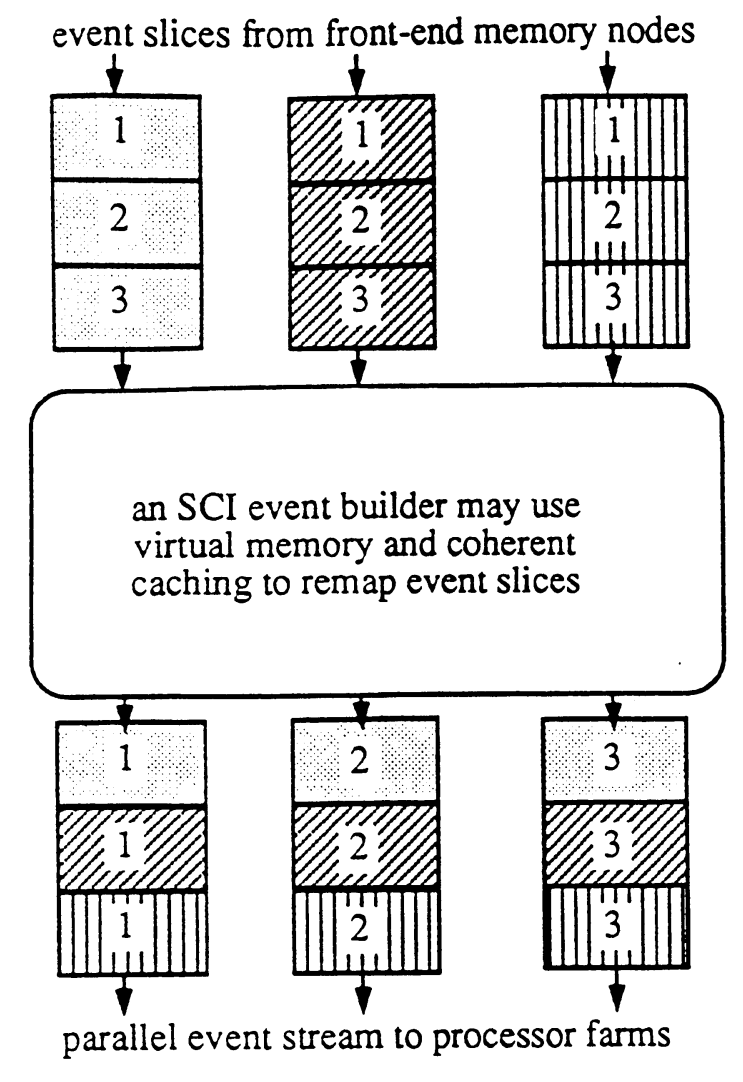

Fig. 1 An event builder produces a parallel output stream of events which are assembled from a parallel input stream of event slices. 
Workstations, which were considered unattractive because they lacked a high bandwidth interface and efficient software, are expected to be equipped with an SCI interface and may therefore become a cheaper alternative to embedded processors.

Front-end memories are located far away from the processors. Instead of designing custom links, an SCI provides a reliable connection with flow control and buffering of data on node chips. Memories may be located far away from the SCI port and even be part of SCI's second-level cache.

\subsection{Architectures}

There are two fundamentally different ways to implement data acquisition systems. In the processor-driven approach, data are read under processor control. In a data driven system, sources write their data in the processors' memory.

In a shared-memory environment, the processor-driven approach has the advantage that data do not have to be copied to the memory of each processor. Data are accessed during the processing when needed. They are also easier to implement in software. The draw back is that the path between a processor and a memory may be long. For fast processors, this can cause intolerable data access latencies, leading to starvation.

In the data-driven approach, sources send all their data at the highest possible speed. Pipelining (sending the next data before the first has arrived) makes this very efficient and eliminates data access latency problems. However, processors may be overrun by data, hence the need for flow control. Processors receive a full copy of the data, all of which are not necessarily processed thus increasing bandwidth requirements.

\section{SIMULATION}

\subsection{Objectives}

The use of simulation as an aid in designing data acquisition systems or to help understand their behaviour is not new. Usually, simulations have been performed with time resolutions typical of the physics event rate. This may be sufficient for a qualitative understanding of the data flow. Parameters which influence the data rate, such as traffic on a bus, are either estimated or extrapolated from measurements. Detailed simulations with a resolution of $\approx 100 \mathrm{~ns}$ are required to understand the available bandwidth between a memory and a processor exchanging 64-byte cache lines.

The simulation should provide insight into the behaviour of data acquisition system topologies (memories, processors and their interconnections), resolve architectural design considerations as discussed in the previous section, and clarify issues concerning data 
flow and resources (how much memory, how many processors, latencies and bandwidth). Simulation is also a valuable tool in the study of SCI protocols.

\subsection{The SCI modelling program}

The SCI modelling program was intended to simulate large LHC-type data acquisition systems containing over 1000 nodes. An accurate simulation of the data flow at a granularity of $100 \mathrm{~ns}$ is now possible with the availability of cheap but high-powered workstations. A provision to simulate at varying time resolutions has been foreseen.

An object-oriented approach has been chosen because it provides excellent modularity. It should be possible in the future to develop independently simulation of other major subsystems (VMEbus, Futurebus+ and Fastbus) and integrate these with minimal effort. Low-level protocols may be hidden in base-level objects from which user modules (such as front-end memories or readout processors) may be derived.

\subsection{Simulation status}

The simulation now includes most of the logical specifications: interconnections (ringlets, SCI-to-SCI bridges), transactions (move, read and write), packets (request, receive and echo), transaction queues, bandwidth allocation and queue acceptance protocols. Not included yet are the cache coherence protocols. Addition of a graphical user interface and animation is foreseen.

The bandwidth allocation and queue acceptance protocols have been implemented in accordance with the spirit of the SCI but are not yet fully conforming to the latest standards proposal. We believe that the discrepancies will not invalidate the results presented in sect. 4 .

\section{LOW-LEVEL SCI PROTOCOLS}

\subsection{Simulation}

To emphasize the effect of different configurations on the system bandwidth, the move 256 transaction has been used in the examples in figs 3 to 6 . This transaction carries 256 bytes of data and has no response. The simulation assumes that nodes can emit or absorb data at "infinite" speed by setting the node interface delays to zero. Performance is strongly influenced by the available amount of buffer space and the number of simultaneously outstanding requests. The first four examples allow only one transaction at a time (it has no influence in this particular configuration with nodes of "infinite" speed).

Simulation results are presented in two plots. The upper one shows the raw and net traffic on each link. "Raw" traffic (no shading) includes packet header, control 
link. The "net" bandwidth (solid shading) indicates the data content of each packet. Echo packets have no data, read transactions have their data in the request packet, write transactions in the response packet. Retry packets are not included. The lower plot shows the net data entering (solid shading) and leaving (no shading) each node.

\subsection{Scalability of rings}

The scalability properties of SCI rings are illustrated by simulation of a simple system containing ten nodes (fig. 2). The most favourable conditions are shown in fig. 3, when all nodes move data to their next neighbour. Request packets traverse one link, the smaller echo packets traverse $\mathrm{n}-1$ links. Each node sends and receives data at a rate close to the maximum bandwidth of a single SCI point-to-point link (nominally, 1 Gbyte/s). The system bandwidth, i.e. the sum of the bandwidths of all links, is almost proportional to $n$, the number of nodes.

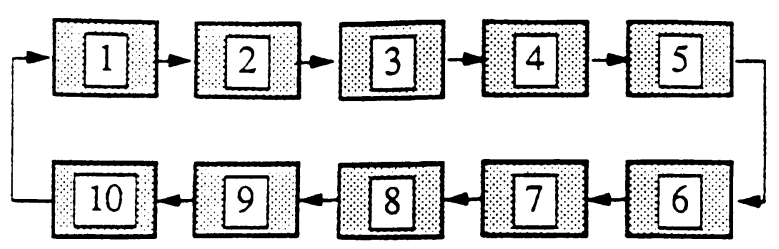

Fig. 2 Simple SCI system consisting of a single ring with ten nodes.

Figure 4 shows the least favourable conditions, when data are moved to previous neighbours. Request packets traverse $\mathrm{n}-1$ links, echo packets one link. The system then behaves as a time-multiplexed bus. The system bandwidth is equal to the maximum bandwidth of a link (1 Gbyte/s) giving a fraction proportional to $1 / \mathrm{n}$ for each node.

Figure 5 shows what happens when each node selects a random target without any flow control. The average path length for all packets is $n / 2$ links, giving a system bandwidth twice that of a single link ( 2 Gbytes/s). Flow control may restrict the system bandwidth in order to ensure fair sharing, as illustrated in fig. 6.

Often read and write operations will be mixed, producing data path lengths of half the ringlet on average. Traffic patterns will be similar to those of figs 5 and 6 . Scalability might therefore be difficult to achieve in general. 


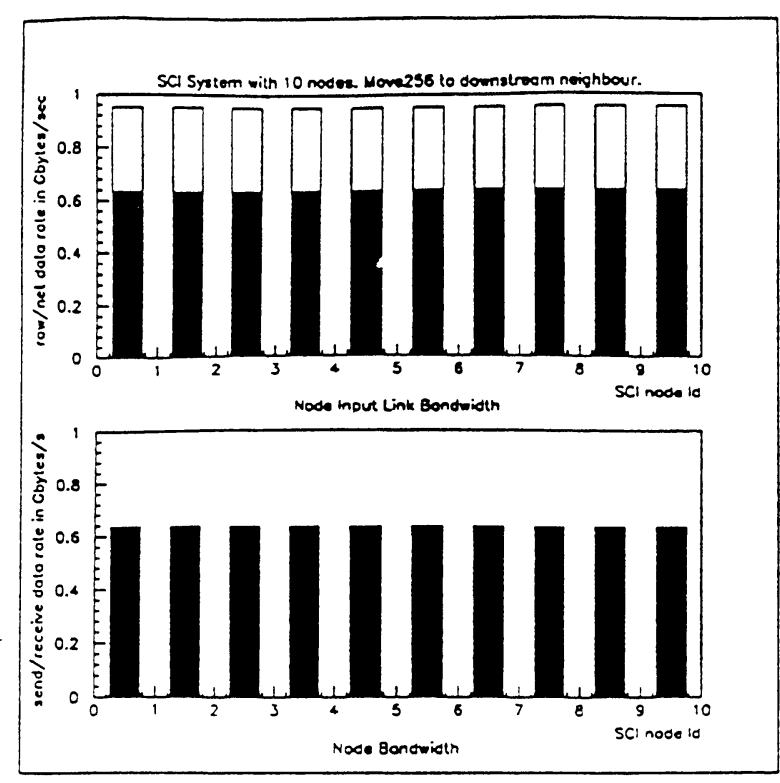

Fig. 3 An SCI ringlet is scalable when data are moved to next neighbours. The system bandwidth is almost proportional to the number of nodes (in this example, $600 \mathrm{Mbytes} / \mathrm{s}$ for each node).

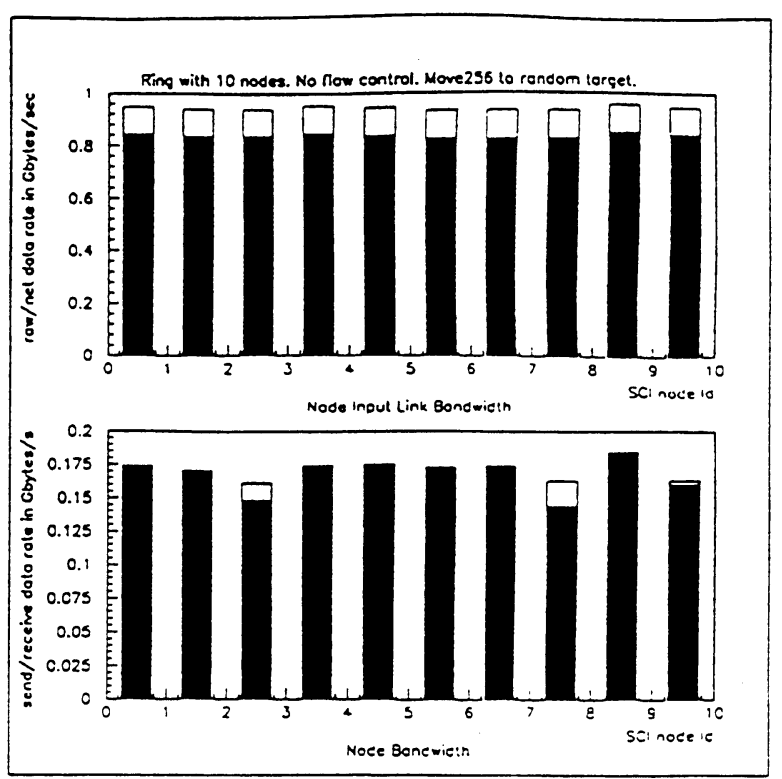

Fig. 5 An SCI ringlet does not scale when each node selects a random target without flow control. The system bandwidth limit is twice that of an equivalent bus (here $\approx 175$ Mbytes/s).

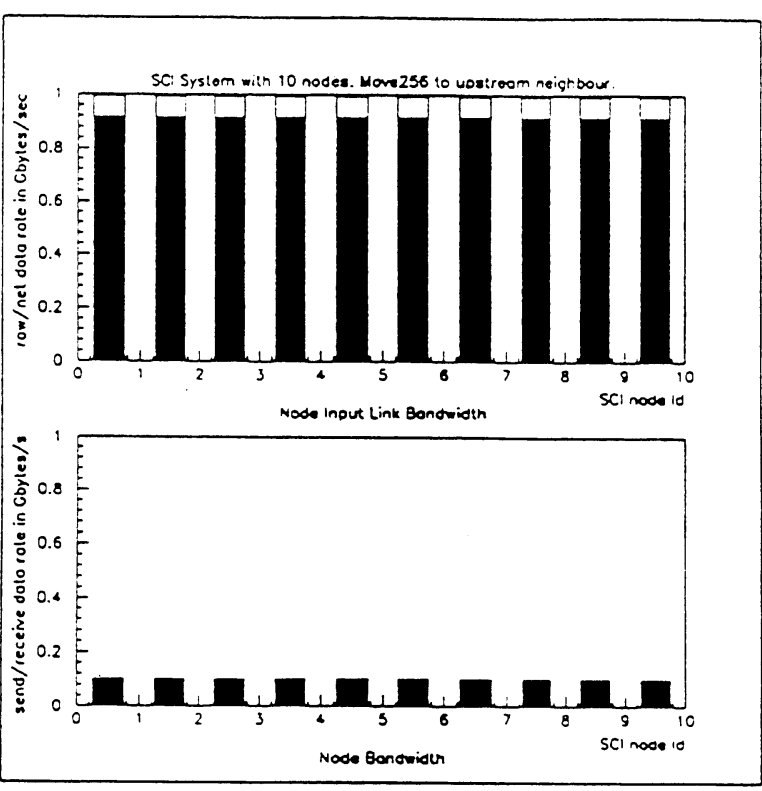

Fig. 4 An SCI ringlet behaves as a "bus" when data move to previous neighbours and do not scale. System bandwidth is independent of the number of nodes (100 Mbytes/s in this example).

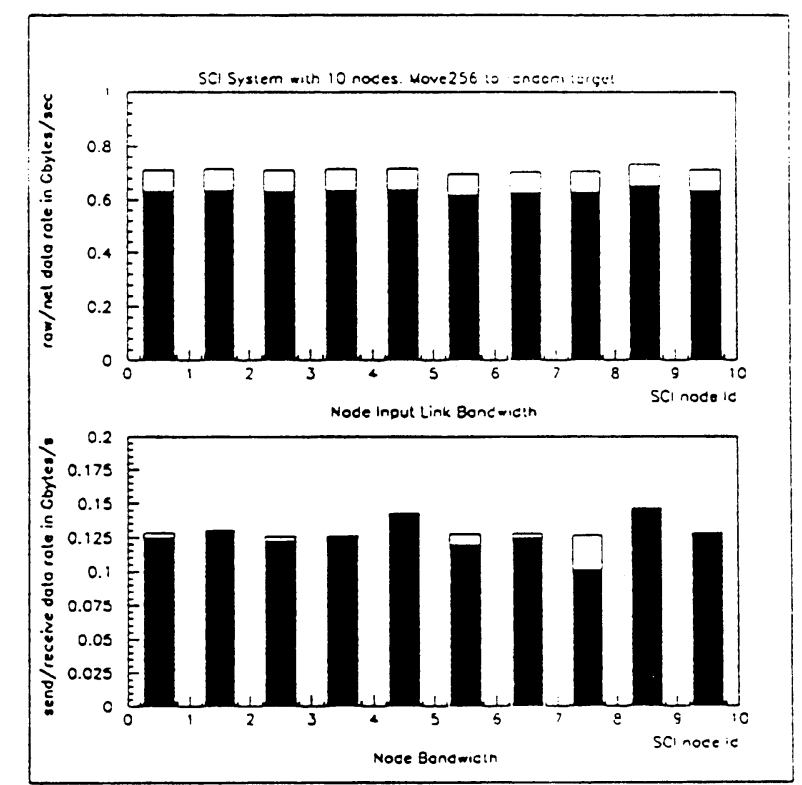

Fig. 6 Flow control protocols guarantee a fair share of the available bandwidth to each node, at the expense of system bandwidth ( $\approx 125 \mathrm{Mbytes} / \mathrm{s}$, as compared to $\approx 175 \mathrm{Mbytes} / \mathrm{s}$ ). 


\subsection{Flow control}

The influence of the bandwidth allocation protocol is even more striking when all nodes on a single ring move data to a unique target, as shown in figs 7 and 8 . Such a configuration is not unlikely for data acquisition systems, e.g. a ringlet of front-end memories with one bridge to the rest of the system. Figure 7 shows nine nodes moving data (move64) to a single target (node 10). The receiving node can buffer 10 packets. Performance does not improve with more than ten buffers, but decreases with less buffer space. Figure 8 is similar, except that move64 has been replaced by write64. The bandwidth allocation protocol prevents the unique target from sending response packets and so gives a surprisingly low throughput (5-10 Mbytes/s). Requesting nodes consume bandwidth by sending packets which are subsequently rejected ("retry" packets). A change to the bandwidth allocation protocol, or higher priority to responses improves the performance. This example illustrates that the bandwidth allocation and queue acceptance protocol do not always co-operate to assure optimal forward progress.

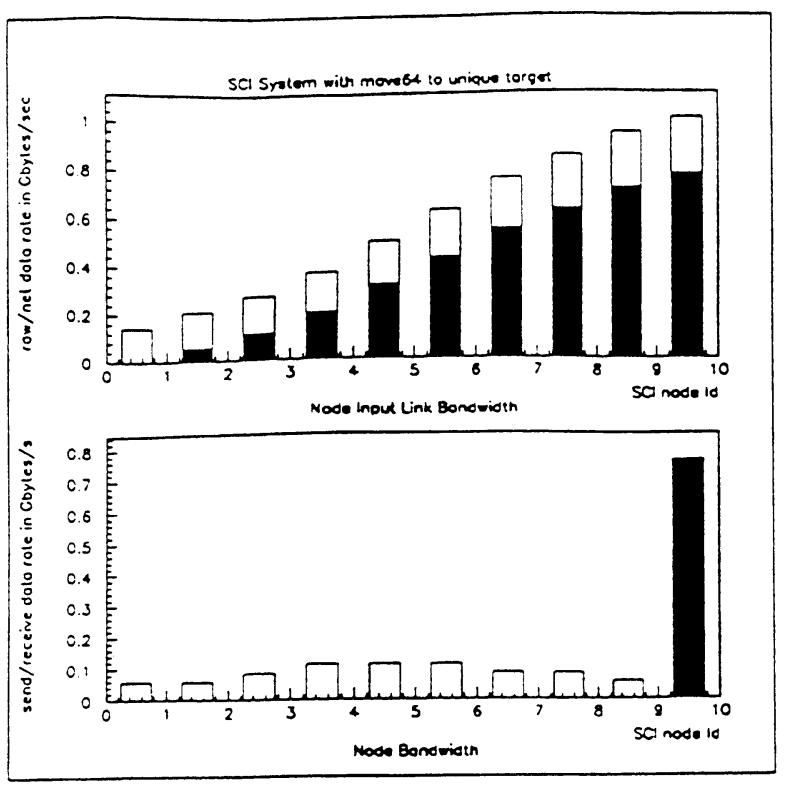

Fig. 7 An SCI ringlet where nine nodes send data to a tenth target using the move64 transaction. The system shows a characteristic "bus"-like behaviour with a bandwidth of $\approx 75$ Mbytes per node. Since there are no response packets, available bandwidth is shared between request packets.

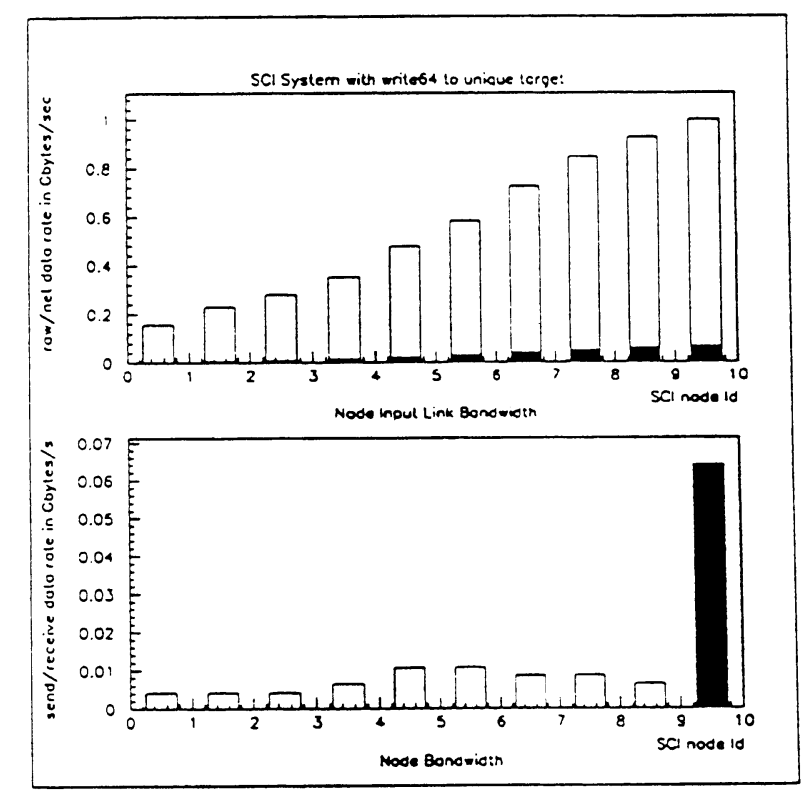

Fig. 8 Replacing the move64 by a write64 transaction changes the system bandwidth dramatically. Response packets from node 10 compete with request packets from nodes $1-9$ for ring access, resulting in poor forward progress. Giving higher priority to responses would improve this.

\subsection{The SCI bridges}

Multiple ring systems are needed for data acquisition applications. The two-ring system of fig. 9 has been simulated with cross traffic over both bridges. In the example, nodes 1 and 6 are bridges. Nodes 2, 3, 4 and 5 send data to nodes 7, 8, 9 and 10 . Similarly, nodes $7,8,9$ and 10 send data to nodes $2,3,4$ and 5 , all using the write 64 
transaction. All nodes provide buffer space for three request and response packets. The results are shown in fig. 10. It can be clearly seen that the bridges are saturated at 1 Gbyte/s. Most of the traffic consists of "retry" packets. The problem is the bandwidth allocation: a bridge "arbitrates" at the same level as other nodes, whilst carrying all data of a ring. Response packets, which have to cross a bridge, compete with local ring traffic (mainly useless retry packets) for access to the ring. The system bandwidth is still $\approx 100 \mathrm{Mbytes} / \mathrm{s}$ per ring, or $25 \mathrm{Mbytes} / \mathrm{s}$ per node. This can be improved by giving higher priority to bridges or switches.

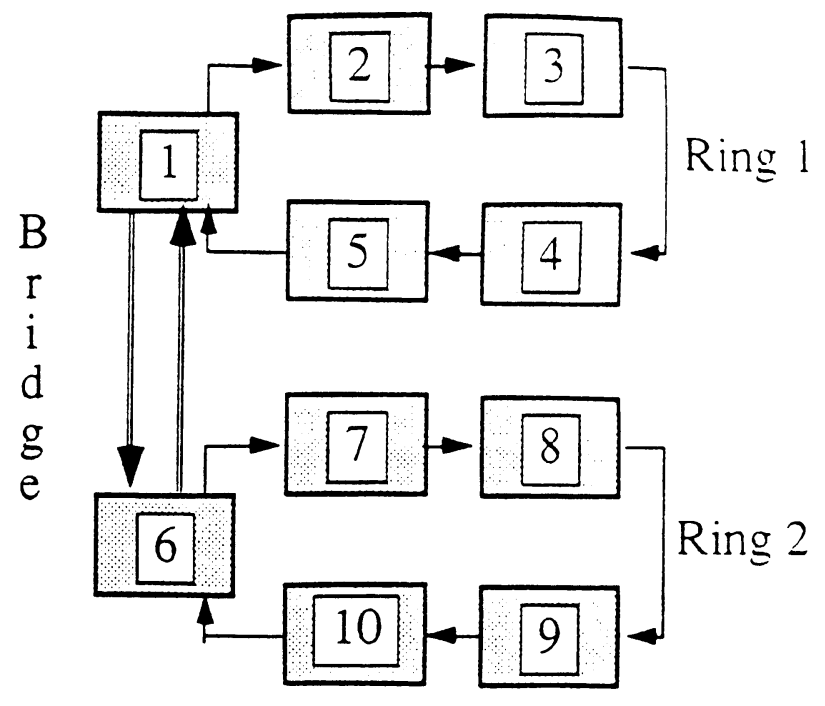

Fig. 9 Bridged SCI system with two rings.

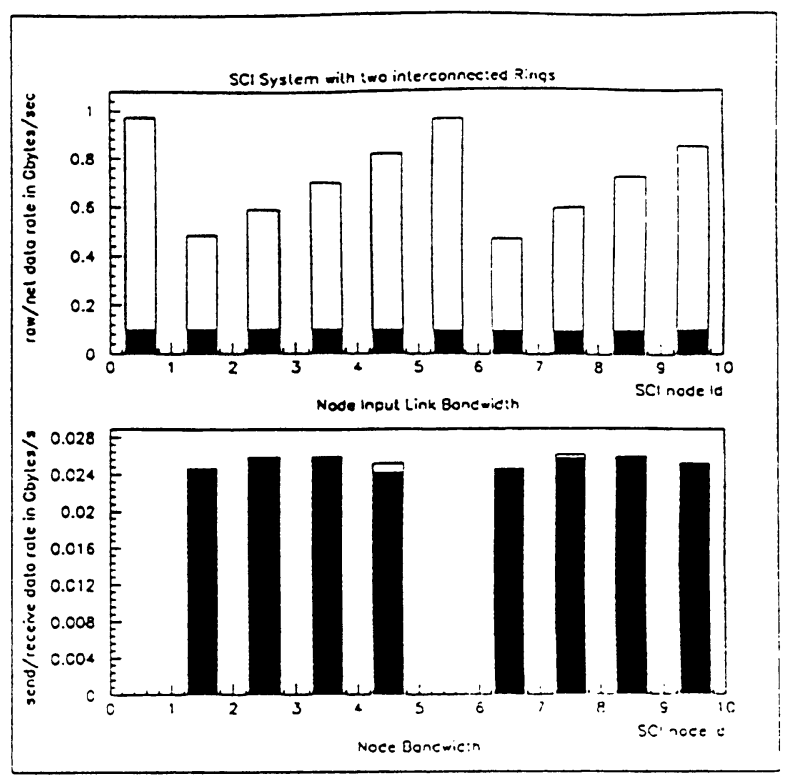

Fig. 10 Cross traffic on an SCI system with two bridges. All nodes on the first ring write to nodes on the second ring, and vice versa. The system bandwidth is clearly limited by the two bridges (nodes 1 and 6). More buffer space in the bridges, combined with unfair bandwidth allocation, could improve the system bandwidth, which is now $\approx 25 \mathrm{Mbytes} / \mathrm{s}$ for each node. 


\section{THE LHC DATA ACQUISITION SYSTEMS}

\subsection{The model}

A general model of an LHC data acquisition system is described in ref. [4]. A possible SCI-based implementation is described in ref. [5] and illustrated in fig. 11. Although the model is only an approximation (detectors and readout electronics are still under development) it is sufficiently realistic to test major architectural choices. There are three detectors: a tracker, a calorimeter and a muon detector. It is assumed that the data acquisition hardware is segmented in $\approx 1000$ independent units which may be thought of as front-end crates containing memories and processors. The units need not to be implemented in SCI, provided that they have an SCI output port (perhaps a dual-port memory, or a bridge to a bus-based system).

\section{Event data in $~ 1000$ units}

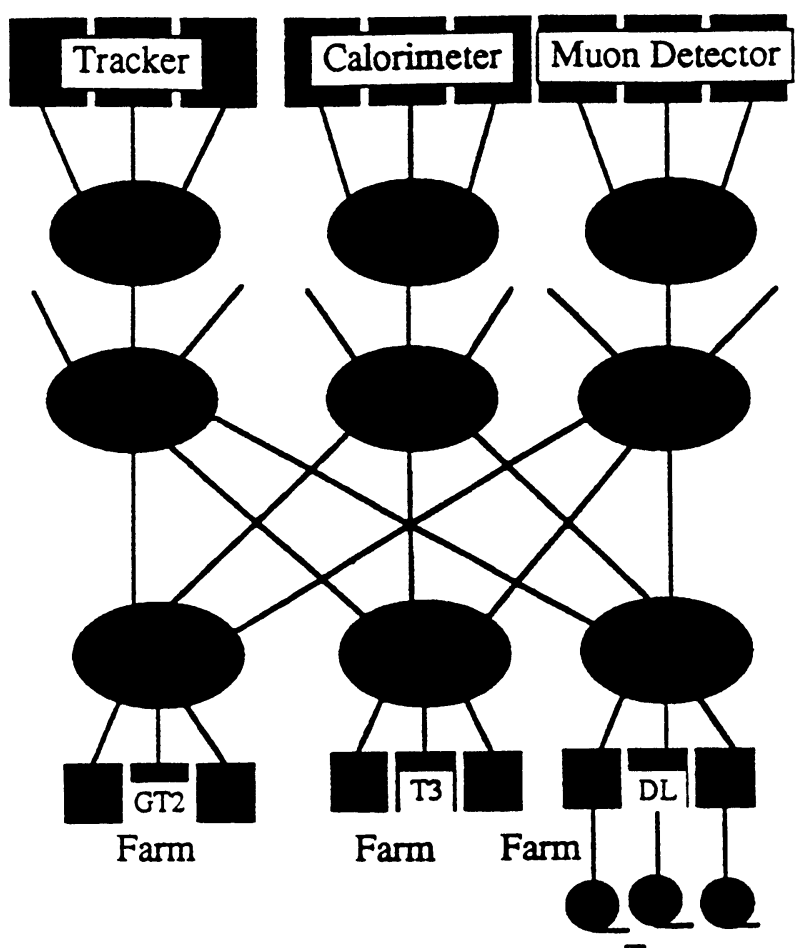

Tapes

Fig. 11 Model of an LHC-based data acquisition system with three detectors (tracker, calorimeter and muon detector). The system consists of $\approx 1000$ front-end data memories and three processor farms: global second-level trigger (GT2), third-level trigger (T3) and data logger (DL). The system is implemented as an SCI system containing over 1000 nodes.

Each unit is collecting data from one sector of a detector; it contributes approximately an equal amount of data and has a uniform interface to the rest of the readout system. Detector channels can be linked in various ways to these units, which may be far away from the detector. Optical fibres will very likely be used for long 
distances. These should be placed between the detector and the units or between memory ports and the first node interface. Detector data are stored in pipelines (in the units) at a rate of $66 \mathrm{MHz}$. Units contain enough storage to buffer events during the trigger decisions.

There are three trigger levels. The first level (T1) is implemented in hardware in the units. The second-level trigger is split into a local part (LT2) requiring data from a single unit, and a global part (GT2) requiring the combined output of the local trigger processors. The LT2 processing is performed in the units GT2 by a farm of SCl-based processors. Data in each unit are compressed and formatted for the next stages of trigger processing and recording in the units just after the LT2 processing. We assume that data may be compressed by a factor ten, e.g. by converting channel values in physical parameters such as track coordinates, momenta and energies. The third-level trigger and data logger will then use compacted data. The third-level trigger T3 is a software decision based on data from a complete event. It is also implemented as an SCI farm similar to the global second-level trigger but with more processing time available. Finally, filtered data are recorded by a third SCI farm DL.

\subsection{Data rates}

Data rates at the different processing stages are illustrated in fig. 12. After $\mathrm{T} 1$, the typical event size is $10^{7}$ bytes at a rate of $10^{5} \mathrm{~Hz}$, that is, $10^{12}$ bytes/s. The second-level trigger needs only $\approx 1 \%$ of these data, $10^{10}$ bytes/s. Distributed between 1000 processors these amounts to $10^{7}$ bytes/s per processor, which is easily reached by standard buses. The output from each local processor is estimated to be $10^{3}$ bytes which gives an aggregate data rate of $10^{8}$ bytes/s into the GT2 processor farm. This exceeds the capacity of existing backplane buses but is within the capabilities of an SCI.

After compression in the units, the data for $\mathrm{T} 3$ and the data logger have an average size of $10^{6}$ bytes at a rate of $10^{3} \mathrm{~Hz}$. Previous experiments have shown that trigger decisions need on average only $10 \%$ of the data. One reason for this is that events may be rejected at an early stage of the analysis when only a small fraction of the data has been examined. This gives a data rate of $10^{8}$ bytes/s to $\mathrm{T} 3$. Event recording requires data to be copied from the unit memories to the data logger, but at a rate of $10^{2} \mathrm{~Hz}$ after the T3 decision. The data recording rate still amounts to $10^{8}$ bytes/s.

In a processor-driven approach the traditional event builder may be avoided because the data are accessed directly. The data-driven approach requires copying data at a rate of $10^{9}$ bytes/s, of which less than two times $10^{8}$ bytes/s is finally used. 


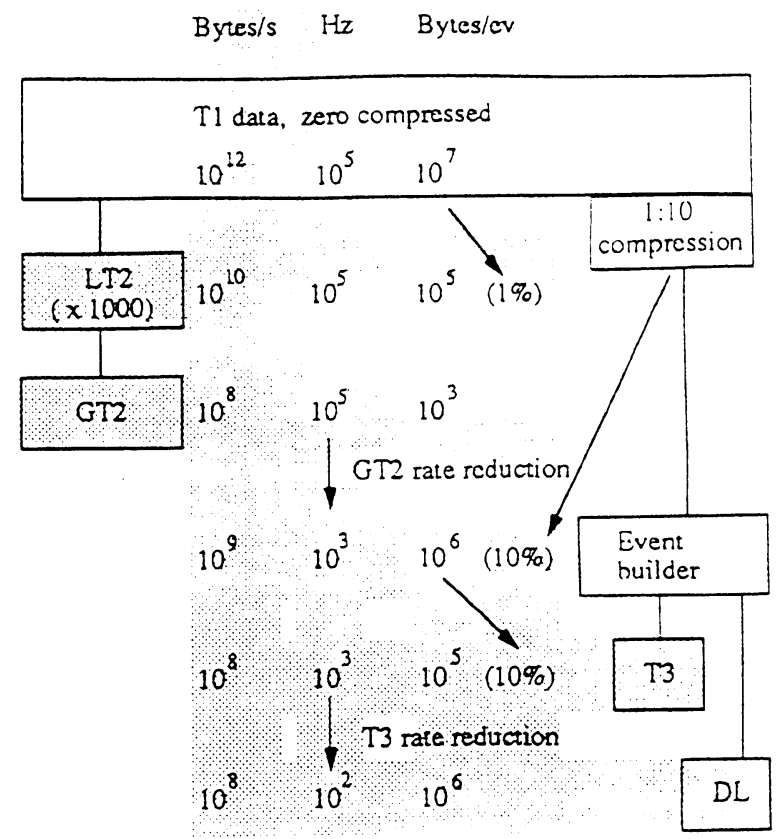

Fig. 12 Data rates for the model of the LHC data acquisition system at the different processing stages. The second-level trigger is distributed amongst a set of local processors (LT2) which contribute to the overall decision taken by the global trigger processor (GT2). After event building, data are further filtered by the third-level trigger processor (T3) and finally recorded by the data logger (DL).

\subsection{Simulation}

As a first exercise the simple data acquisition architecture illustrated in fig. 11 has been simulated using the processor-driven approach. The three different detectors (tracker, calorimeter and muon detector), are each represented by 320 front-end memory nodes. These memories are grouped in rings containing 20 nodes and connected to detector rings via a bridge. There are three detector rings, each containing 16 bridges to front-end memory rings and three bridges to processor farm rings (GT2, T3 and DL).

The total number of nodes in the system is 1083 , as summarized in table 1 . All processors belonging to each of the three farms read the data directly from front-end memories at the highest possible speed, using the SCI read64 transaction. The purpose of the simulation is to investigate the feasibility of an SCl-based system providing enough bandwidth and CPU capacity to sustain a data rate of at least $100 \mathrm{Mbytes} / \mathrm{s}$ for each of the three data streams. Simulation results are shown for farms of three processors each. 
Table 1 SCI simulation parameters

\begin{tabular}{|ll|}
\hline Detectors & 3 (tracker, calorimeter and muon detector) \\
SCI rings & 57 \\
Processor farms & $3(\mathrm{GT} 2, \mathrm{~T} 3$ and DL) \\
Front-end memories & 960 \\
SCI bridges & 114 \\
Processors & 9 (three for each farm) \\
Total number of nodes & $1083(960+114+9)$ \\
Node delay & $12 \mathrm{~ns}$ (includes cable) \\
Bridge delay & $12 \mathrm{~ns}$ (bridge cable) \\
Interface delay & $200 \mathrm{~ns}$ (memory latency) \\
\hline
\end{tabular}

\subsection{Results}

"Hot spots" in the system can immediately be identified from fig. 13, which shows the data rate on the SCI links which interconnect the nodes. There are three peaks showing 200 Mbytes for each of the three detector rings (tracker, calorimeter and muon detector). More detail is visible in fig. 14, where nodes 1015-1083 are plotted. This shows from left to right: a front-end memory ring (nodes 1026-1046), the muon detector ring (nodes 1047-1065) and the three farms GT2 (nodes 1066-1071), T3 (nodes 1072-1077) and DL (nodes 1078-1083). The peak raw data rate for the processor farms is also $200 \mathrm{Mbytes} / \mathrm{s}$; the net data rate is $150 \mathrm{Mbytes} / \mathrm{s}$. The data rate for the front-end memory rings is much lower ( $\approx 0.5 \mathrm{Mbytes} / \mathrm{s}$ for a memory). It peaks at $10 \mathrm{Mbytes} / \mathrm{s}$ for the bridge which carries all the trigger data away. The total amount of data transported over the system is 20 Gbytes/s (raw) or 10 Gbytes/s (net).

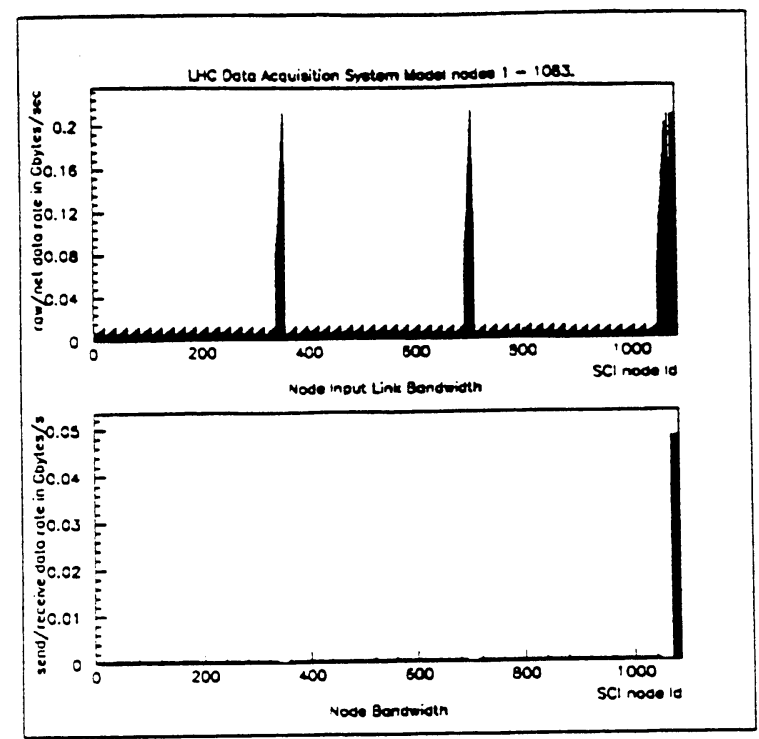

Fig. 13 Simulation of the SCI-based model of an LHC data acquisition system containing 1083 nodes. The plot at the top shows the data traffic on the interconnections. "Hot spots" can easily be identified. The plot at the bottom shows the processor bandwidth. 
The amount of data flowing in or out of these nodes (with the exception of bridges) is also shown. Figure 14 indicates that each processor belonging to one of the farms GT2, T3 or DL receives a net amount of $50 \mathrm{Mbytes} / \mathrm{s}$, resulting in $150 \mathrm{Mbytes} / \mathrm{s}$ for each processor farm. Varying the number of processor elements for each farm between one and six scales well: the bandwidth of each processor decreases only slightly from $55 \mathrm{Mbytes} / \mathrm{s}$ to $45 \mathrm{Mbytes} / \mathrm{s}$ (not shown).

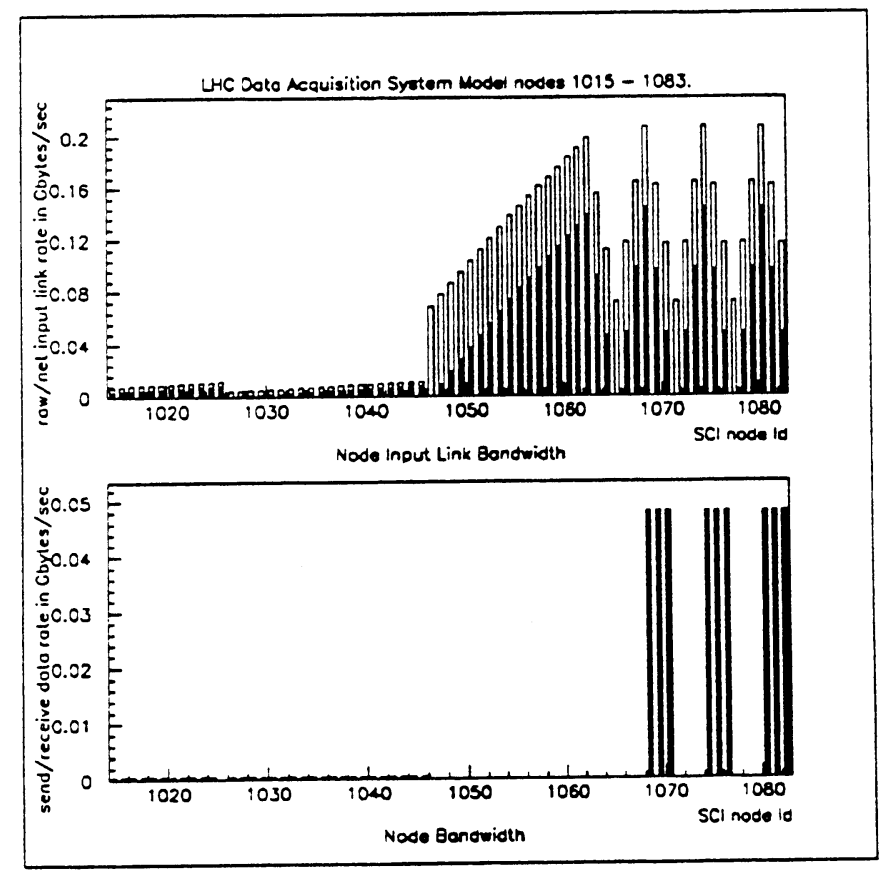

Fig. 14 More detail is visible in this enlargement, showing from left to right: a front-end memory ring, the muon detector ring and the three processing farms (GT2, T3 and DL).

\subsection{Discussion}

The throughput of the processor-driven system is determined by the data access latency and the number of simultaneous requests. Each request from the processor results in the transfer of 64 bytes. The latency is the time it takes to complete a transaction. The throughput is given by the product of the data size (64 bytes) and the number of simultaneous requests divided by the latency.

The simulation allowed only one request at a time. The observed average latency was 1330 ns per packet of 64 bytes. This is in good agreement with the data rate ( 50 Mbytes/s corresponds to a latency of $20 \mathrm{~ns} / \mathrm{byte}$ ). The minimum latency can be calculated by adding all node, bridge and interface delays between processors and memories plus the packet delay (raw packet size in symbols multiplied by $2 \mathrm{~ns}$ ). The parameters of table 1 give a value of 1300 ns. The small difference with the value obtained from the simulation indicates that contention is very low. 
The parameters used are based on short distances between nodes. Long distances to the detectors can be covered in two ways: either optical fibre SCI links which will increase latencies perhaps compromising the desired data throughput or non-SCI fibres between the detectors and the front-end units. The latter solution would require a large number of fibres carrying small amounts of data but keep latencies low.

Performance of modern RISC processors is adversely affected by data access latencies (a cache miss may cause the loss of several hundred CPU instructions). Manufacturers are looking for general solutions to this problem. One way of improving CPU hardware is the introduction of cache prefetch (such as the MC88110). Compilers need to be changed to exploit this.

Scalability with this topology is not assured once the "hot spots" reach saturation. A different network, probably containing more levels, would be required at the expense of increased latencies. This effect can be diminished by using pipelined bridges or completely suppressed by switches.

\subsection{Future research}

Alternatives need to be investigated, in particular, the data-driven approach. This will require a vast amount of computing power (the more economical processor driven model ran for ten hours on a DECstation 5000 to simulate $10 \mathrm{~ms}$ ). Higher-level protocols extended to software, event and data structures and realistic triggers must be included in the simulation. The use of virtual memories, cache memories and their interface to applications through system software, has to he studied. To obtain realistic rates one should simulate the software in the processors which will ultimately determine the data rate.

\section{Acknowledgements}

We would like to thank S. Cittolin, P. Ponting and E.M. Rimmer from ECP division at CERN for their interest, encouragement and material support. Thanks also to our CERN colleagues for their expert assistance in making available the considerable computer power required for the simulations. 


\section{REFERENCES}

[1] P1596 WG of the Microprocessor Standards Committee, SCI logical, physical and cache coherence specifications, P1596/D1.00, 23 January 1991, draft for the Sponsor Ballot Review, New York: IEEE (1991).

[2] ISO/IEC JTC1 SC26 WG8, The VICbus specification, draft version 1.1, ed. C.F. Parkman (May 1991).

[3] N. Ellis, S. Cittolin and L. Mapelli, Signal processing, triggering and data acquisition, proc. of the Large Hadron Collider Workshop, Aachen, vol. I (1990) 505-538.

[4] H. Müller et al., Buses and standards for LHCs, proc. of the Large Hadron Collider Workshop, Aachen, vol. III (1990) 159-164.

[5] J.F. Renardy et al., SCIs at LHCs, proc. of the Large Hadron Collider Workshop, Aachen, vol. III (1990) 165-169. 